Please do not remove this page

RMIT

UNIVERSITY

\title{
Two-dimensional crystallization of hard sphere particles at a liquid-liquid interface
}

Marnette, Olivier; Perez, Eric; Pincet, Frederic; Bryant, Gary

https://researchrepository.rmit.edu.au/esploro/outputs/9921864183901341/filesAndLinks?institution=61RMIT_INST\&index=null

Marnette, O., Perez, E., Pincet, F., \& Bryant, G. (2009). Two-dimensional crystallization of hard sphere particles at a liquid-liquid interface. Colloids and Surfaces A, 346(1-3), 208-212.

https://doi.org/10.1016/j.colsurfa.2009.06.018

Document Version: Accepted Manuscript

Published Version: https://doi.org/10.1016/j.colsurfa.2009.06.018

Repository homepage: https://researchrepository.rmit.edu.au

(C) 2009 Elsevier B.V. All rights reserved.

Downloaded On 2023/04/27 00:50:57 +1000

Please do not remove this page 
Thank you for downloading this document from the RMIT Research Repository.

The RMIT Research Repository is an open access database showcasing the research outputs of RMIT University researchers.

RMIT Research Repository: http://researchbank.rmit.edu.au/

\section{Citation:}

Marnette, O, Perez, E, Pincet, F and Bryant, G 2009, 'Two-dimensional crystallization of hard sphere particles at a liquid-liquid interface.', Colloids and Surfaces A, vol. 346, no. 1-3, pp. 208-212.

See this record in the RMIT Research Repository at:

http://researchbank.rmit.edu.au/view/rmit:4426

Version: Accepted Manuscript

Copyright Statement: (c) 2009 Elsevier B.V. All rights reserved.

Link to Published Version:

http://dx.doi.org/10.1016/j.colsurfa.2009.06.018 


\title{
Two dimensional crystallization of hard sphere particles at a liquid- liquid interface.
}

Olivier Marnette ${ }^{\mathrm{a}}$, Eric Perez ${ }^{\mathrm{a}}$, Frederic Pincet ${ }^{\mathrm{a}}$ and Gary Bryant ${ }^{\mathrm{b} *}$

a'Laboratoire de Physique Statistique de l'Ecole Normale Supérieure, UMR8550 Associée aux Universités Paris VI et Paris VII, 24, rue Lhomond, 75231 Paris Cedex 05, France

${ }^{\mathrm{b}}$ Applied Physics, School of Applied Sciences, RMIT University, Melbourne, Vic 3000, Australia

\section{* Corresponding Author}

A/Prof. Gary Bryant

Applied Physics, School of Applied Sciences

RMIT University

GPO Box 2476V

Melbourne, 3001

Tel: 61-3-99252139

Fax: 61-3-99255290

Email: gary.bryant@rmit.edu.au

\begin{abstract}
A method for studying crystallization of hard-sphere like particles in two dimensions is presented. The method involves trapping the particles at the interface between two immiscible liquids. Particles at the interface undergo 2D Brownian motion, and at sufficiently high densities crystallization is observed. The pseudo hard-sphere nature of the particle interactions under these conditions is maintained, as demonstrated by the area density at which crystallization occurs. In contrast to established techniques for studying crystallization in pseudo 2D hard spheres, the particles trapped at the interface undergo no vertical motion, so the system is in principle closer to a true $2 \mathrm{D}$ system. The method is therefore amenable to the studying the effects of polydispersity on crystallization behaviour. The advantages and disadvantages of the method are discussed.
\end{abstract}

Keywords: 2D crystallization, colloids, polydispersity, hard-spheres 


\section{Introduction}

Since the initial work over two decades ago [1], interest in the phase behaviour of hard sphere suspensions has increased markedly, with many hundreds of papers published. Colloidal suspensions of hard spheres are excellent model systems for the study of the fundamental processes of crystallization and glass formation. The primary advantage of colloidal suspensions over atomic and molecular systems is that the time scales for crystallization are orders of magnitude slower, and the length scales are orders of magnitude larger, enabling much higher temporal and spatial resolution. Apart from the differences in time and length scales, one of the major differences between crystallization in atomic systems and in colloidal suspensions is that any colloidal suspension contains a range of particle sizes around a mean value - ie they are polydisperse. Much of the earlier work largely ignored the effects that polydispersity plays in the phase behaviour of colloidal suspensions. Recently, however, polydispersity effects on colloidal crystallization in three dimensions have attracted attention both in theory and simulation [2-4] and in experimental studies [5-11]. The understanding that has emerged from these studies is that polydispersity has a dramatic effect on the kinetics of phase transitions in three dimensions, as well as on the crystalline structure.

There has also been a considerable amount of interest in phase transitions in 2D systems particularly with regard to nanotechnology applications (e.g. $[12,13])$. Despite this interest, the effect of polydispersity in two dimensions has received little attention. The only studies to date are: the simulations of Santen and Krauth [14], which suggested the possibility of a 2D glass at high polydispersities; those of Pronk and Frenkel [15], who produced a phase diagram for polydisperse hard disks showing that the miscibility gap does not increase with polydispersity, in contrast to the 3D system; and those of Frydel and Rice [16] which showed that the presence of polydispersity gives rise to an effective interaction. However, to the knowledge of the authors there are no experimental studies of the effects of polydispersity on crystallization in 2D. A number of two dimensional systems have been studied experimentally, including studies of charged particles trapped at the air/water interface [17], particles with short range paramagnetic repulsions [18-20], and studies of charged particles confined between glass plates [21]. However polydispersity is unlikely to be a significant issue for such systems with ranged interactions. The most extensive experimental studies of quasi two dimensional systems of hard spheres are those of Rice and colleagues (e.g. [22-25]), using particles confined between glass plates. Despite the elegance and utility of the work using this method, it is not optimal for studying the effects of polydispersity. First, in this system the constraining glass plates have a separation which is $\sim 20 \%$ greater than the particle diameter, meaning that the particles retain a limited degree of vertical motion. Second, there are the complicating effects of the proximity of the confining walls $[23,24]$. Third, and most importantly, from the perspective of the current work, if there are particles of different sizes present, the vertical position of their mid-planes may be significantly different, and smaller particles can potentially become trapped above (or below) the mid planes of the larger ones at high densities.

In this paper we present an experimental approach which is amenable to the study of the effects of polydispersity on the crystallization of hard spheres. The proposed method involves trapping particles at the interface between two immiscible liquids. The principle is that the particles are suspended in the top liquid, and fall under gravity onto the interface with a denser, immiscible liquid below. Even though the particles are denser than the lower liquid, they are trapped at the interface of the two liquids by the interfacial tension. This interfacial tension is sufficiently large that vertical motions due to thermal fluctuations are insignificant compared to the particle size, while lateral motions remain Brownian. The method has in principle several advantages: first, there are no wall effects; second, as there is no possibility of significant vertical motion, the system is much closer to being a true 2 
dimensional system, which becomes most important for polydisperse and multi-modal systems; third, once gravitational settling has occurred the particle density will remain constant if the interface is flat, however if the interface has a small curvature, the system can be slowly compressed (or expanded). This enables the possibility of studying the 2D system during compression. In this paper we describe the system, discuss the difficulties and assumptions, and demonstrate that the particles retain their pseudo hard-sphere nature.

\section{Materials and Methods}

The HS particles are made up of polymethylmethacrylate (PMMA), sterically stabilised with polyhydroxy stearic acid (PHSA) and exhibit hard sphere behaviour [26, 27] in suitable solvents (eg decalin). The particles used here have a mean radius $\mathrm{R}$ of $1.36 \mu \mathrm{m}$ and a polydispersity of $4.9 \%$ [5]. In order to develop a method of trapping the particles at a liquid-liquid interface, whilst retaining their hard sphere character, the liquids must be carefully chosen. Decalin (density 0.89 g. $\mathrm{cm}^{-3}$, viscosity 2.6 $\mathrm{mPa} . \mathrm{s}\left(20^{\circ} \mathrm{C}\right)$ ) is chosen for the upper liquid, as this is a standard solvent used for 3D studies of hard spheres, and is well characterized. The lower liquid must be immiscible with decalin, but allow the hard sphere character of the particles to be retained. Ideally the density of the lower liquid should be greater than that of the particles. However, even if the density is slightly smaller, the interfacial tension at the interface may be sufficient to trap the particles. Finally, the second liquid should have a low dielectric constant to minimize the meniscus at the contact line between the particle and the liquids.

The liquid used in this study is 1-3 propanediol, (density of $1.05 \mathrm{~g} . \mathrm{cm}^{-3}$, viscosity $39.2 \mathrm{mPa} . \mathrm{s}\left(20^{\circ} \mathrm{C}\right)$ ). Although the particle density $\left(1.22 \pm 0.03 \mathrm{~g} . \mathrm{cm}^{-3}\right)$ is above the propanediol density, it still allows the particles to be trapped at the interface because of the interfacial tension. The lower density of decalin allows the particles to sediment to the interface. The cell used to anchor particles at the liquid/liquid interface is shown in Fig. 1. The circular cell consists of two sections of different diameter, with a shoulder at a $90^{\circ}$ angle to which the interface between the liquids can anchor. Using this approach, the shape of the interface, whether planar, spherical or otherwise, can be controlled with good precision, regardless of the wetting properties of the cell material. Both chambers have an inlet port for injecting the liquids, and the top chamber has an outlet port for air to escape. Thin glass plates are attached to the cell at the top and bottom to seal the cell and allow optical observation.

Prior to an experiment the cell is cleaned by sonication with detergent, rinsed with ultrapure water (18.2 M $\Omega$ - Elgastat maxima) and dried. Propanediol (Merck, for synthesis), is injected into the lower chamber with a syringe connected via a flexible tube. Care is taken to avoid air bubbles in the tubes which may be difficult to remove. In general, the surface is curved and it can be made planar by accurately adjusting the volume of injected liquid. Sufficient decalin is then injected through the upper inlet in order to completely fill the cell and remove any air bubbles. The interface is much less visible than the free surface of propanediol, making it impossible to determine the planarity by eye. To roughly check the planarity of the interface, a grid is observed through the cell - if the grid is not deformed, the surface is planar to first order (the planarity is ultimately determined by observing the particles at the interface with the microscope).

Once the interface is approximately planar, both tubes are sealed to fix the volume of the liquids. A small volume of a concentrated suspension of particles is injected through the air outlet using a syringe needle inserted into the middle of the decalin layer, just above the centre of the interface. The air outlet is then sealed, and the system is placed on a microscope stage. Although particles begin to sediment immediately, it takes several hours for all the particles to reach the interface and about one day to have a stable distribution of particles at the interface. Assuming the microscope and cell are horizontal, and 
the liquid volumes have been well adjusted, then one obtains an interface of approximately $30 \mathrm{~mm}$ diameter, approximately flat to within $\sim 30 \mu \mathrm{m}$. In all cases particles were trapped at the interface, with particles never observed in the lower liquid.

The observation of the two dimensional hard sphere system is done with a Leica inverted microscope with a magnification of $x 16$. The focussing is adjusted to have the particles appear as bright spots in the microscope. The centre of the bright spots always has a Gaussian intensity profile. Images from the microscope were recorded with a webcam (1280x960 pixels, pixel size 5.6x5.6 $\mu \mathrm{m}$, Philips PCVC750K). The images provided by the CCD camera were analysed by detecting local intensity maxima on the bright spots of the image through Gaussian fits using Matlab codes (Matlab 7 and image processing toolbox). More extensive details of the experimental procedure and the analysis can be found in Marnette [28].

The surface fraction occupied by the particles is the parameter which controls the phase behaviour of two dimensional hard sphere systems. For these experiments it is taken as the number of particles in the field of view multiplied by the mean projected area of a particle, divided by the field of view area. The uncertainty at low magnification is dominated by the number of particles missed by the image analysis and the uncertainty of the mean particle size. Comparisons between manual and automatic counting lead to an overall uncertainty of about $2 \%$ in area fraction.

The simplest measure which can be used to characterise ordering in a two dimensional hard sphere system is the pair correlation function $\mathrm{g}(\mathrm{r})$ which gives the probability of finding a particle at a distance $\mathrm{r}$ from a reference particle:

$$
g(\|\vec{r}\|)=\frac{\langle d N(r)\rangle}{\rho_{o} 2 \pi r d r}
$$

where $\rho_{o}$ is the mean surface density of particles, $\langle d N(r)\rangle$ is the number of particles whose centres are located within a ring of radius $r$ and thickness $\mathrm{dr}$, averaged over all the considered particles. The function is obtained from the list of the positions of all the particles at the interface. The list of all the interparticle distances is calculated from one image. Then, the pair correlation function is calculated for interparticle distances $0<r<r_{\max }$ with a resolution dr. $r_{\max }$ is the maximum interparticle separation used in the calculation of $\mathrm{g}(\mathrm{r})$. Only the particles which are sufficiently central, ie. which are located at a distance from the edge larger than $r_{\max }$, can be used to calculate $g(r)$ with their neighbours. For the results presented here $r_{\max }=25 \mathrm{R}$, and $\mathrm{dr}=0.05 \mathrm{R}$. Pair correlations calculated for each central particle are added and normalised to obtain $\mathrm{g}(\mathrm{r})$.

\section{Results \& Discussion}

Figure 2 shows images of 2D particles in the fluid phase and the crystalline phase, along with the radial distribution functions, $g(r)$, calculated from each image. While the top image appears to have some local order, this does not correspond to crystallinity, as shown by the $g(r)$, which decays to the noise after around 5 times the average inter-particle distance (indicated by the circles on the image). The bottom image on the other hand shows large crystalline regions, separated by well defined grain boundaries. The observation of grain boundaries is a simple and robust criterion to affirm that a system is in the crystalline state. The size of the crystalline grains is a good indicator of the length of the correlations of crystalline order. This is confirmed by the $g(r)$, which shows that the $2^{\text {nd }}$ peak is split (one of the indicators of crystallinity). This splitting of the second peak will be used hereinafter as the 
indicator of crystallinity. In addition, the peaks go out to about 20 inter-particle distances, which corresponds approximately with the size of the crystals, as indicated by the circle on the image.

Having established that the particles can crystallize on the interface, it now needs to be determined if the hard sphere character of the interactions is maintained. While the interactions cannot be measured directly, the nature of the interactions can be inferred by determining the area fraction at which crystallization is observed, and comparing with the theoretical value.

Figure 3 shows the $g(r)$ measured at a number of different area fractions between $50 \%$ and $80 \%$. The figure on the left demonstrates that long range order begins to appear only above $70 \%$. As shown on the expanded scale on the right, for densities above $72 \%$ the second peak shows a clear splitting. Using the position of the first peak as a calibration, the vertical lines show the theoretical peak positions as functions of area fraction. The solid lines indicate the positions of the fluid peaks (at integral values of the first peak), whereas the dotted lines represent the positions of the additional hexagonal crystalline peaks. Theory predicts freezing at an area fraction of $69 \%$ and melting at an area fraction of $72.4 \%$. As can be seen in figure 3, there is excellent correspondence between the theoretical lines and the crystalline peaks above $75 \%$, while there is no evidence of these peaks at the lowest area fractions. At area fractions around $69 \%$ the $\mathrm{g}(\mathrm{r})$ hints at the beginnings of crystallinity. This area fraction is at the border of the coexistence region, within the uncertainty in the area fraction.

The rationale for the development of the system described here is to allow for the measurement of the effects of polydispersity in 2D. In order to establish that this system is capable of such measurements, a few issues need to be addressed.

First, how large are the particle fluctuations normal to the interface? These fluctuations can be estimated by calculating the strength of the surface energy barrier, as described by Pieranski [17]. This analysis shows that the energy associated with a particle of radius $\mathrm{R}$ trapped at an interface is:

$$
\mathrm{E}=2 \pi \gamma_{\mathrm{pl}}\left(\mathrm{R}^{2}-\mathrm{Rz}\right)+2 \pi \gamma_{\mathrm{pu}}\left(\mathrm{R}^{2}+\mathrm{Rz}\right)-\pi \gamma_{\mathrm{ul}}\left(\mathrm{R}^{2}-\mathrm{z}^{2}\right)
$$

Where $\gamma_{\mathrm{ij}}$ are the interfacial tensions, and the subscripts $\mathrm{p}, 1$ and $\mathrm{u}$ refer to the particles, lower and upper liquids respectively, and $\mathrm{z}$ is the height of the centre of the particle above the interface. Differentiating this twice with respect to $z$ gives the effective spring stiffness of the interface, $k=2 \pi \gamma_{\mathrm{ul}}$. So a particle trapped at the interface will be subject to a restoring force with an energy of:

$$
\mathrm{E}=\frac{1}{2} \mathrm{kx}^{2}=\pi \gamma_{\mathrm{ul}} \mathrm{x}^{2}
$$

For the interfacial tension here $\left(\gamma_{\mathrm{ul}} \sim 14 \mathrm{mN} / \mathrm{m}\right)$, a fluctuation of order $1 \mathrm{kT}$ will result in a vertical displacement of only $\mathrm{x}=0.3 \mathrm{~nm}$. So vertical motion due to thermal fluctuations is negligible in this system.

Second, do particles of different size sit at different heights at the interface? Measurements of the dynamics of individual particles (at low densities) show 2D Brownian behaviour. By determining the effective viscosity at the interface, relative to the viscosities of the two liquids, it is found that $80 \%$ of each particle is in the top liquid (for more details see Marnette [28]). Clearly, smaller particles will have their mid-plane at a lower point than larger particles. How significant is this effect? 
The height of the particle mid-plane relative to the interface is given by [17]:

$\mathrm{Z}=\frac{\gamma_{\mathrm{pl}}-\gamma_{\mathrm{pu}}}{\gamma_{\mathrm{ul}}} \mathrm{R}=\mathrm{cR}$

where $\mathrm{c}$ is a constant. The difference in height between the two particles of different size (subscripts L (Large) and S (Small)) would be:

$\Delta \mathrm{z}=\mathrm{c}\left(\mathrm{R}_{\mathrm{L}}-\mathrm{R}_{\mathrm{S}}\right)$

The individual surface tensions are not known. To estimate the value of $\mathrm{c}$, from the measurements above we assume that $80 \%$ of the particle height is in the top liquid, which yields a value of $\mathrm{c} \sim 0.6$. Note that it may be more appropriate to use the volume or cross sectional area, but both approximations would yield smaller values of $\mathrm{c}$, and therefore a smaller effect. The value $\mathrm{c} \sim 0.6$ therefore represents an upper limit of the effect. For the particles used here, $(\mathrm{R}=1.36 \mathrm{~mm}$, polydispersity $4.9 \%), 95 \%$ of particles are between 1.22 and $1.49 \mu \mathrm{m}$. For these extremes $\Delta \mathrm{z} \sim 0.16 \mu \mathrm{m}$. This situation is illustrated in figure 4 (drawn to scale). As can be seen from the figure, the effective radius of the smaller particle, $\mathrm{R}_{\mathrm{eff}}$, is slightly smaller than $\mathrm{R}_{\mathrm{S}}$. From geometry it is easy to show that:

$\mathrm{R}_{\mathrm{eff}}=\sqrt{\mathrm{R}_{\mathrm{S}}^{2}-\Delta \mathrm{z}^{2}}$

For the values here this yields $\mathrm{R}_{\mathrm{eff}}=1.21$ (compared with $\mathrm{R}_{\mathrm{s}}=1.22$ ). Thus the difference in height is insignificant for polydispersities of order $\sim 5 \%$, and is not very sensitive to the exact value of $\mathrm{c}$.

The final consideration is the contribution of effects which could give rise to behaviour which is not hard sphere in nature, including charging effects and capillary interactions. The particles are sterically stabilized and top liquid is non-polar, so charging effects are expected to be very small. Dipolar effects, if present, would result in a repulsion which would cause crystallization to occur at lower area fractions than is observed here [17]. For particles of similar size and density, floatation forces lead to an attraction between particles [29]. However, these forces are negligible for particle radii less than 5-10 $\mu \mathrm{m}$ [29]. Moreover, if significant attractions were present, one would expect particles to become trapped in attractive wells, losing their Brownian character. This is not observed here. Thus, while such interactions may exist, they appear to be smaller than the thermal energy, and so do not significantly affect the two dimensional phase behaviour.

These results show that the system does indeed have behaviour consistent with that of a 2D hard sphere system. There may be deviations from hard sphere behaviour too subtle to be ascertained using the present methods. Other methods for characterizing the interactions, including the use of other criteria for determining crystallinity, such as bond order parameters [25], as well as detailed comparisons with other pseudo 2D hard sphere systems, are left for future work.

Finally, it should be noted that the technique has a number of inherent difficulties. First, optical resolution is low, due to the fact the light passes through multiple interfaces and two layers of liquids, and the particles and liquid have similar refractive indices. In addition, at high densities it is inherently difficult to determine particle positions automatically when they are virtually touching. The use of confocal microscopy combined with fluorescent core particles [30] would significantly improve the resolution. Second, in most experiments there is significant collective drift at all but the highest densities, caused by convective motions in the liquid. This is largely caused by heating due to the 
illuminating beam, and can be reduced by observing using light filtered through a visible light filter (green). Again confocal microscopy may reduce these effects. Third, obtaining a completely flat interface at the micron level is virtually impossible. However, this also has advantages - the curvature can be used to compress the particles into the centre, or allow them to expand. With careful experiments it may be possible to study the compression and expansion of this system.

\section{Conclusions}

We have demonstrated an alternative experimental system for studying crystallization of 2D hard spheres. This system confines these spheres at the interface between two liquids chosen to approximately maintain the hard-sphere character of the particles. We have shown that the spheres crystallize at approximately the theoretically predicted density, and that the particles undergo two

dimensional Brownian motion. Using this system, studies of the effects of polydispersity on crystallization in $2 \mathrm{D}$ may be possible.

Acknowledgements: The authors would like to thank Werner Krauth, whose theoretical work inspired this study, and for useful discussions during the design of the project. We would also like to thank Bill van Megen for preparing the particles. This project is proudly supported by the International Science Linkages established under the Australian Government's innovation statement, Backing Australia's Ability and the French-Australian Science and Technology program (FAST).

\section{References}

[1] P.N. Pusey, W. van Megen, Phase Behaviour of Concentrated Suspensions of Nearly Hard Colloidal Spheres., Nature (London) 320 (1986) 340-342.

[2] D.A. Kofke, P.G. Bolhuis, Freezing of Polydisperse Hard Spheres., Physical Review E 59 (1999) 618-622.

[3] P. Bartlett, Freezing in Polydisperse Colloidal Suspensions, Journal of Physics: Condensed Matter 12 (2000) A275-A280.

[4] S. Auer, D. Frenkel, Suppression of Crystal Nucleation in Polydisperse Colloids due to Increase of the Surface Free Energy., Nature (London) 413 (2001) 711-713.

[5] H.J. Schöpe, O. Marnette, W. van Megen, G. Bryant, Preparation and characterization of particles with small differences in polydispersity, Langmuir 23 (2007) 11534-11539.

[6] H.J. Schöpe, G. Bryant, W. van Megen, Effect of polydispersity on the crystallization kinetics of suspensions of hard-spheres when approaching the glass transition, Journal of Chemical Physics 127 (2007) 084505.

[7] H.J. Schöpe, G. Bryant, W. van Megen, Small changes in particle-size distribution dramatically delay and enhance nucleation in hard sphere colloidal suspensions., Physical Review E 74 (2006) 060401(R).

[8] H.J. Schöpe, G. Bryant, W. van Megen, Two-step crystallization kinetics in colloidal hardsphere systems, Physical Review Letters 96 (2006) 175701.

[9] S. Martin, G. Bryant, W. van Megen, Crystallization kinetics of polydisperse colloidal hard spheres II: Binary mixtures., Physical Review E 71 (2005) 021404.

[10] S. Martin, G. Bryant, W. van Megen, Observation of a Smecticlike Crystalline Structure in Polydisperse Colloids., Physical Review Letters 90 (2003) 255702.

[11] S. Martin, G. Bryant, W. van Megen, Crystallization kinetics of polydisperse colloidal hard spheres: Experimental evidence for local fractionation., Physical Review E 67 (2003) 061405.

[12] K.C. Grabar, R.G. Freeman, M.B. Hommer, M.J. Natan, Preparation and Characterization of Au Colloid Monolayers, Analytical Chemistry 67 (1995) 735-743. 
[13] N.D. Denkov, O.D. Velov, P.A. Kralchevsky, I.B. Ivanov, H. Yoshimura, K. Nagayama, Mechanism of Formation of 2-Dimensional Crystals from Latex Particles on Substrates, Langmuir 8 (1992) 3183-3190.

[14] L. Santen, W. Krauth, Absence of thermodynamic phase transition in a model glass former., Nature (London) 405 (2000) 550-551.

[15] S. Pronk, D. Frenkel, Melting of polydisperse hard disks, Physical Review E 69 (2004) 066123.

[16] D. Frydel, S.A. Rice, Phase diagram of a quasi-two-dimensional colloid assembly, Physical Review E 68 (2003) 061405.

[17] P. Pieranski, Two-dimensional interfacial colloidal crystals., Physical Review Letters 45 (1980) 569-572.

[18] K. Zahn, J.M. Méndez-Alcarez, G. Maret, Hydrodynamic Interactions May Enhance the SelfDiffusion of Colloidal Particles, Physical Review Letters 79 (1997) 175-178.

[19] K. Zahn, G. Maret, Dynamic Criteria for Melting in Two Dimensions, Physical Review Letters 85 (2000) 3656-3659.

[20] K. Zahn, R. Lenke, G. Maret, Two-Stage Melting of Paramagnetic Colloidal Crystals in Two Dimensions, Physical Review Letters 82 (1999) 2721-2724.

[21] C.A. Murray, D.H. Van Winkle, Experimental observation of two-stage melting in a classical two-dimensional screened Coulomb system, Physical Review Letters 58 (1987) 1200-1203.

[22] H. Diamant, B. Cui, B. Lin, S.A. Rice, Correlated particle dynamics in concentrated quasi-twodimensional suspensions, Journal of Physics-Condensed Matter 17 (2005) S4047-S4058.

[23] P. Karnchanaphanurach, B. Lin, S.A. Rice, Melting transition in a quasi-two-dimensional colloid suspension: Influence of the colloid-colloid interaction, Physical Review E 61 (2000) 40364044.

[24] B.H. Lin, J. Yu, S.A. Rice, Direct measurements of constrained Brownian motion of an isolated sphere between two walls, Physical Review E 62 (2000) 3909-3919.

[25] A.H. Marcus, S.A. Rice, Phase transitions in a confined quasi-two-dimensional colloid suspension, Physical Review E 55 (1997) 637-656.

[26] G. Bryant, S.R. Williams, I.K. Snook, Q. LinMao, E. Perez, F. Pincet, How Hard is a Colloidal "Hard Sphere" Interaction?, Physical Review E 66 (2002) 060501(R).

[27] S.M. Underwood, J.R. Taylor, W. van Megen, Sterically Stabilized Colloidal Particles as Model Hard Spheres., Langmuir 10 (1994) 3550-3554.

[28] O. Marnette, Une expérience de vidéomicroscopie pour suivre la crystallisation d'un systéme colloïdal 2D de sphères dures polydisperses sur interface, Université Paris VI, Paris, 2007, p. 195.

[29] P.A. Kralchevsky, K. Nagayama, Capillary forces and structuring in layers of colloid particles., Advances in Colloid \& Interface Science 85 (2000) 145-192.

[30] R.P.A. Dullens, M. Claesson, D. Derks, A. van Blaaderen, W.K. Kegel, Monodisperse CoreShell Poly(methyl methacrylate) Latex Colloids, Langmuir 19 (2003) 5963-5966. 


\section{Figures}

Figure 1 : Schematic of the cell used to confine a hard sphere system in two dimensions. Adjusting the level of the lower liquid allows control of the shape of the interface.

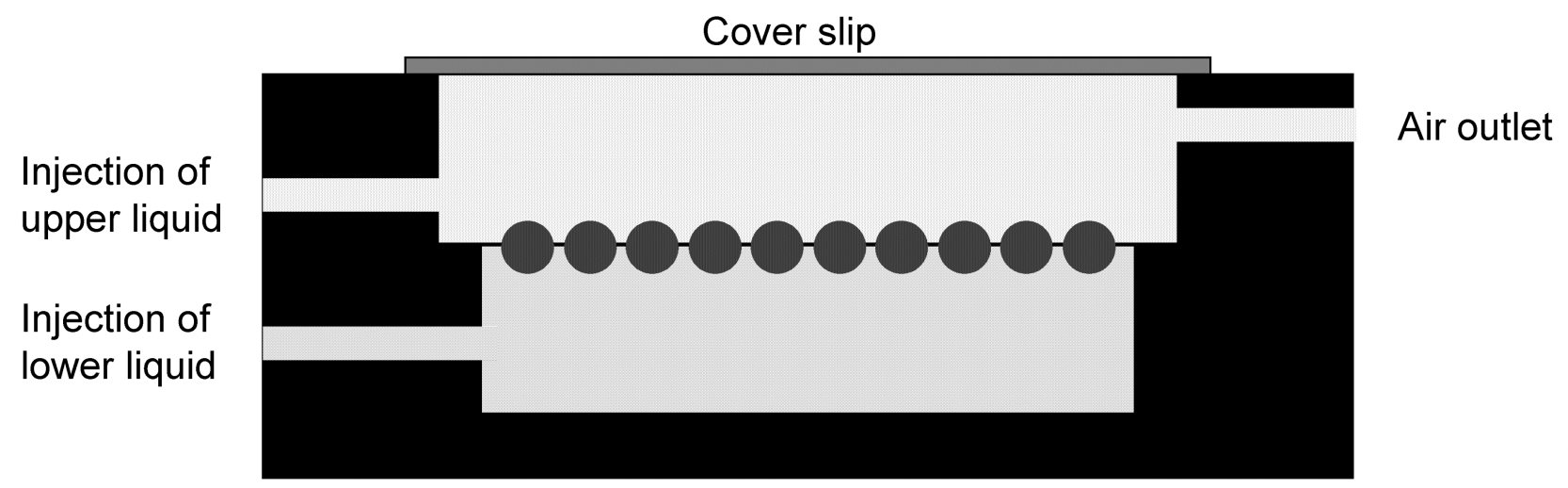

Figure 2: Typical images of a liquid (top) and a poly-crystal (bottom), of SMU23 particles ( $\mathrm{R}=1.36 \mu \mathrm{m}$, polydispersity $=4.9 \%$ ) on a decalin/propanediol interface. Images are at $16 \mathrm{x}$ magnification. The corresponding radial distribution functions, $g(r)$, are shown on the right.

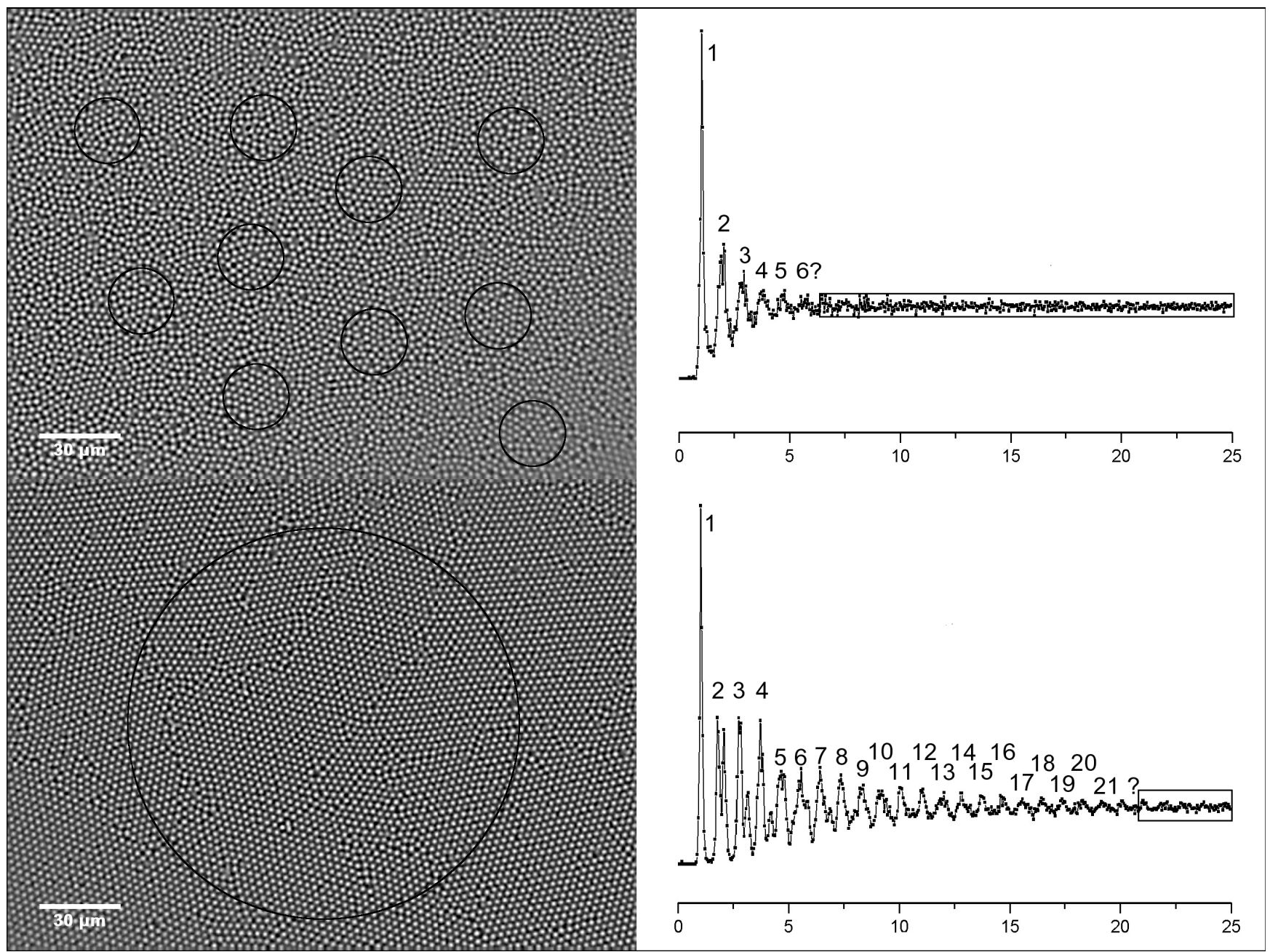


Figure 3: $g(r)$, normalized by the average particle diameter, for several area fractions between $50 \%$ and $85 \%$. The figure on the left is $\mathrm{g}(\mathrm{r})$ out to 25 particle diameters, and the figure on the right is shown on an expanded scale (out to 10 diameters). The theoretical values of the area fractions for freezing $(69.0 \%)$ and melting $(72.4 \%)$ are shown as dotted horizontal lines. The vertical lines represent the positions of the ideal non-compact hexagonal crystal as a function of area fraction. The solid lines correspond to the peaks in $g(r)$ in the fluid phase, and the dotted lines to those peaks which only occur in the crystalline phase.
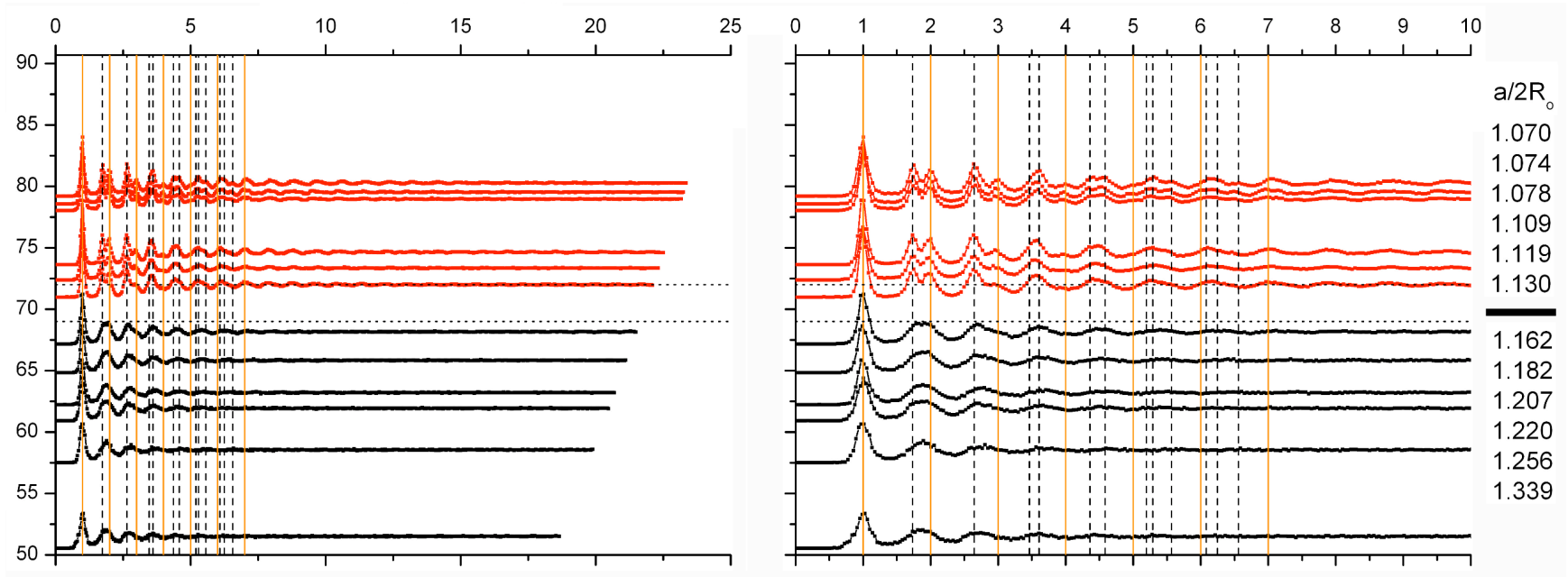

Figure 4: Schematic showing particles of different size floating at different heights. Schematic is drawn to scale for particles at the extremes of the particle size distribution used here (1.49 and $1.22 \mu \mathrm{m}$, encompassing $95 \%$ of the particles), with $\mathrm{c}=0.6$. Symbols are defined in the text.

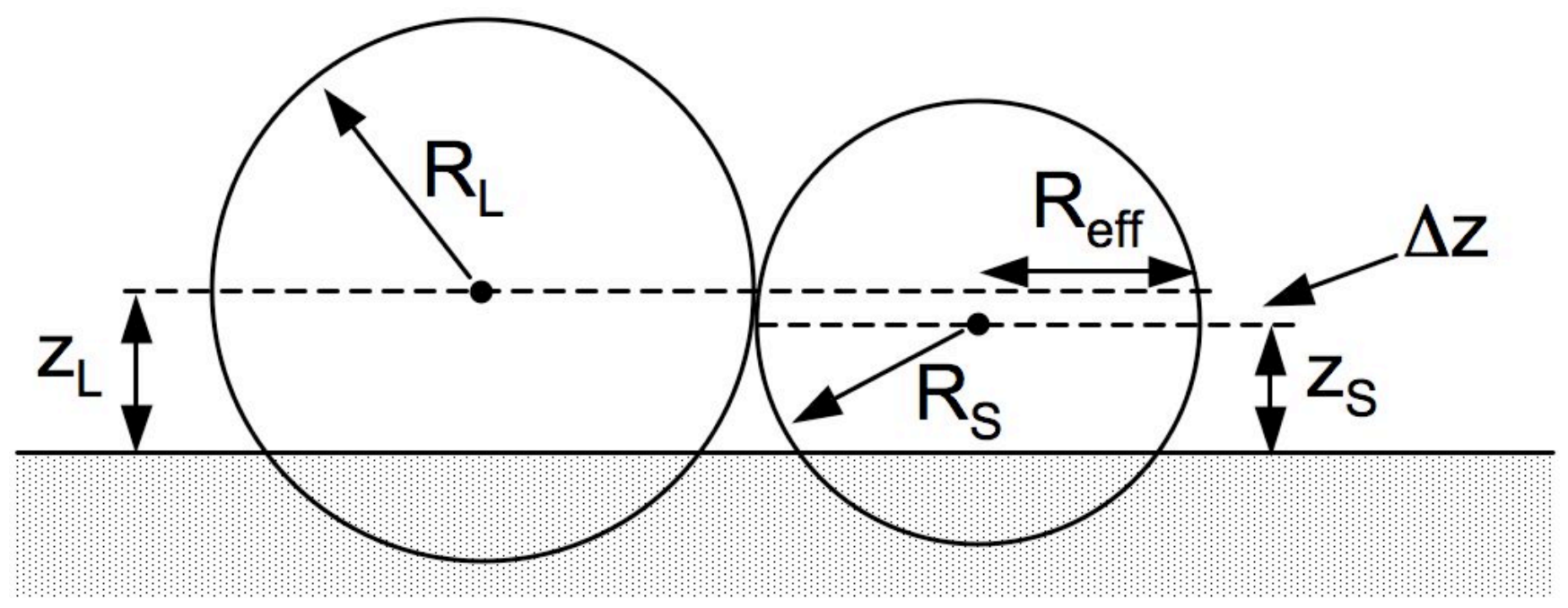

